\title{
Análisis de los factores morfológicos, bioquímicos, fisiológicos y biomecánicos que influyen en el tiro al aro en movimiento
}

Analysis of the morphological, biochemical, physiological and biomechanical factors that influence the shooting of the moving ring

MSc. Eugenio Rodolfo González Pérez. ${ }^{1} \&$ Lic. Aracelia González Paneque. ${ }^{2}$

Recibido: 14-07-2018 / Revisado: 15-08-2018 / Aceptado: 16-09-2018 / Publicado: 04-10-2018

DOI: https://doi.org/10.33262/anatomiadigital.v1i3.1054

\begin{abstract}
.
In Basketball the most used shot is the shot with one hand on the shoulder jumping. In its initial position the ball is held with two hands at the waist and then by means of an impulse of the legs and pendulum of arms, the player jumps up and brings the ball to the initial position to execute the shot with one hand on the shoulder, then when he is at the highest point of the flight the player throws the ball into the basket in the same way as when he takes this shot from the spot, but considering that the shot itself is executed without the
\end{abstract}

\section{Resumen.}

En el Baloncesto el tiro más usado es el tiro con una mano sobre el hombro saltando. En su posición inicial la bola se sostiene con dos manos junto a la cintura y luego mediante un impulso de las piernas y péndulo de brazos, el jugador salta hacia arriba y lleva la bola hasta la posición inicial para ejecutar el tiro con una mano sobre el hombro, después cuando se encuentra en el punto más alto del vuelo el jugador tira la bola al cesto en igual forma que cuando realiza este tiro desde el lugar, pero considerando que el propio tiro se ejecuta sin la participación de las piernas, por

\footnotetext{
1 Facultad de Ciencias Médicas de Mayabeque, Departamento Cultura Física, Mayabeque, Cuba, ergonzales@infomed.sld.cu iD https://orcid.org/0000-0002-7353-0166

2 Facultad de Ciencias Médicas de Mayabeque, Mayabeque, Cuba, agonzalez@infomed.sld.cu https://orcid.org/0000-0002-7353-0167
} 
participation of the legs, therefore the arm extension should be stronger and faster.

Keywords: Basketball, morphological, biochemical, physiological, biomechanical factors. tanto la extensión de los brazos debe ser más fuerte y más rápida.

Palabras claves: Baloncesto, factores morfológicos, bioquímicos, fisiológicos, biomecánicos.

\section{Introducción.}

El Baloncesto, es un deporte de equipo jugado normalmente en pista cubierta, en el que dos conjuntos o equipos de cinco jugadores cada uno, intentan anotar puntos o canastas lanzando una pelota, de forma que descienda a través de las dos cestas o canastas suspendidas por encima de sus cabezas en cada extremo de la pista. El equipo que anote más puntos, convirtiendo tiros de campos o desde la zona de tiros libres, gana entonces el partido. Producto de su dinamismo, espectacularidad y también a la frecuencia de acciones anotadas, es uno de los deportes que posee mayor número de espectadores y participantes en el mundo.

Historia y Desarrollo del Baloncesto en Güines antes del triunfo de la Revolución.

El Baloncesto comienza a practicarse en la Villa de Güines a finales de 1921, de forma muy rudimentaria y con condiciones tanto materiales como técnicas bastantes elementales. La práctica de dicho deporte se efectuaba en los predios del llamado Güines Sport Club, que se encontraba en la calle Habana entre Arango y 5.

Posteriormente comienza a practicarse el Baloncesto en el terreno del Colegio Americano, logrando un buen desarrollo y auge entre el alumnado y los seguidores del mismo.

Ya por el año 1928, se comenzó a practicar Baloncesto en el terreno de la Sociedad Güinera Liceo de Güines que posteriormente sería la Plaza más fuerte de dicho deporte en la localidad.

En 1928 se forma una selección de Güines para enfrentar al equipo del Central Merceditas, partido en el cual Livio Morales Garriz conformó dicho equipo, causando admiración y sensación. Dicho jugador logra ser incluido en el equipo Cuba en varias ocasiones, participando en competencias internacionales como atleta destacado.

En la década del 30, incursiona en el Baloncesto Güinero las féminas, con el equipo del Colegio Americano.

A principios de la década del 30 se celebran en la Habana los Segundos Juegos Deportivos Centroamericanos y Güines se ve representado en el equipo Nacional de Baloncesto masculino por el atleta Livio Morales Garriz. 
A finales de 1935 las muchachas del Colegio Americano mostraban cierto adelanto en el deporte de las canastas, dando buenas demostraciones ante equipos de más nivel y experiencia.

A principios de la década del 40 el Baloncesto había decaído un poco en la afición local, pero estaba resurgiendo de forma vigorosa bajo la hábil y experta dirección de un consagrado del tabloncillo, el Dr. Francisco Ortega Castellanos.

En el mes de Noviembre de 1944 un nutrido grupo de jóvenes del colegio americano de esta Villa celebraron un juego con el equipo de Madruga, donde obtuvieron la victoria 44 x 12 .

También culminando esta etapa vamos a referirnos al equipo nombrado los Sapos del Colegio Americano, los cuales recorrieron la isla de victoria en victoria poniendo el nombre de Güines en las páginas gloriosas del Baloncesto cubano.

Ya en la década de 1950 comienza a efectuarse Campeonatos Infantiles. En 1951 la Villa de Güines participa en el Campeonato Juvenil y Júnior de la Unión Amateur de Cuba. En el año 1953 participando en un tope juvenil el equipo del Liceo de Güines venció al Club de los Hermanos Maristas.

En el año 1955 el equipo de Baloncesto de Güines realiza un viaje a los Estados Unidos para efectuar varios juegos de Baloncesto.

A la llegada del equipo güinero de Estados Unidos se efectuó un juego entre los equipos del Liceo de Bejucal y el Casino Español de Güines. La década del 50-60 fue muy rica en calidad y participación por parte del equipo güinero del Liceo de Güines y otros equipos de la localidad, pero hay que destacar que a pesar de la calidad exhibida por los jugadores de la Villa de Güines siempre la misma fue representada por los Clubes o Sociedades privadas, siendo limitadas las capas sociales humildes.

Historia y Desarrollo del Baloncesto en Güines después del triunfo de la Revolución.

Con el triunfo de la Revolución se produce un cambio radical en la economía, política y social, también el deporte sufre transformaciones, eliminando los clubes y sociedades privadas, al igual que las clases sociales, produciéndose la irrupción en el campo deportivo de las capas humildes de la Villa de Güines y de toda la isla de Cuba.

Muchos de los atletas del Municipio de Güines emigran a los Estados Unidos, y entonces surge el inicio y desarrollo de una nueva generación deportiva. Se inaugura el Coliseo Deportivo de Güines, que ha sido la cuna del desarrollo de muchos baloncestistas güineros.

Por aquel entonces al frente del deporte en el Municipio de Güines Roberto Pérez, destacado deportista de su equipo y gran promotor del deporte güinero y él mismo cursó una invitación 
a los equipos de Baloncesto de los Estados Unidos Trotamundos de Harlen y una selección de la NBA

Estos equipos se encontraban en la Habana efectuando encuentros de exhibición entre ellos y el objetivo de la visita era el desarrollo del Baloncesto y su motivación. Estos juegos fueron de mucha ayuda al Baloncesto güinero, ya que se desarrolló un auge tremendo y mucho entusiasmo por el deporte de las canastas.

Pedro Chappé, uno de los más grandes jugadores de Baloncesto de Cuba representó el Pabellón Internacional de nuestra Patria en múltiples ocasiones y posteriormente

Director de nuestra Selección Nacional de Baloncesto, daba sus primeros pasos en el deporte y le gustaba la forma aguerrida de jugar del equipo de Güines, además por aquella época se creó un equipo femenino que no solo impulsó el Baloncesto femenino en Güines, sino en la región entera. Algunas de las pioneras del Baloncesto revolucionario en Güines fueron:

Guillermina Esquivel, Amelia Báez, Mirta Herrera, Heidi Paret (Nenita)

También ya había germinado la semilla, existiendo una generación de niños de 12 y de 13 años, que le darían desarrollo al Baloncesto en Güines.

En 1963 comienzan los Juegos Nacionales Escolares, existiendo en Güines un gran desarrollo no tan solo en Baloncesto, sino en diferentes deportes. También se creó la Escuela para Profesores de Educación Física Manuel Fajardo, más la creación de los Juegos Escolares Nacionales el auge y desarrollo del Baloncesto güinero era tremendo, ya que el mismo se practicaba en la mayoría de las escuelas, tanto Primarias, como secundarias y Preuniversitarias en el campo, aumentando el número de escuelas en al municipio y la cantidad de alumnos que practiquen el Baloncesto.

El Municipio de Güines, presentaba equipos en todas las categorías y sexos a nivel escolar, así como en la Primera y Segunda Categoría. También alcanzó logros en los Juegos de los trabajadores.

Considero que el auge y la participación del Baloncesto en Güines es buena, la calidad también y esto lo corrobora la ubicación alcanzada por nuestro Municipio en diferentes torneos y justas.

\section{Desarrollo}

Tiro: En el deporte de Baloncesto si el pase es la base, entonces podemos decir que el tiro es la finalidad del juego. Por tanto, el tiro ocupa una posición determinante entre una serie de elementos técnicos de este deporte. El tiro se realiza con una y dos manos, desde el lugar, 
desde distintas posiciones iniciales, desde el hombro, de pecho, por arriba, por abajo y de arriba-abajo.

Tiro en Suspensión: En el Baloncesto el tiro más usado es el tiro con una mano sobre el hombro saltando. En su posición inicial la bola se sostiene con dos manos junto a la cintura y luego mediante un impulso de las piernas y péndulo de brazos, el jugador salta hacia arriba y lleva la bola hasta la posición inicial para ejecutar el tiro con una mano sobre el hombro, después cuando se encuentra en el punto más alto del vuelo el jugador tira la bola al cesto en igual forma que cuando realiza este tiro desde el lugar, pero considerando que el propio tiro se ejecuta sin la participación de las piernas, por tanto la extensión de los brazos debe ser más fuerte y más rápida.

Músculos del tren Superior.

Musculatura Escapulo humeral: Este grupo garantiza la interacción e independencia entre la escápula y el húmero, además actúan como verdaderos ligamentos de la articulación escapulo humeral. En el deporte podemos señalar dos subgrupos, escapulo clavícula humeral o superficial escapulo tuberal o profundo, estos están compuestos por: el músculo subescapular, supraespinoso, infra espinoso, deltoides, redondo menor y mayor, además el coracobraquial.

Músculos del Brazo y del Antebrazo.

Vamos a resumir los músculos de acuerdo al lugar de origen e inserción para su mejor comprensión y estudio.

Relación escapular: Músculo bíceps braquial y cabeza larga del tríceps braquial.

Relación humerocubital: Músculo tríceps braquial (vastos interno y externos), músculo braquial, ánconeo.

Relación humero cubito radial: atronador cuadrado, palmar mayor, primer radia.

Articulaciones que intervienen y su clasificación.

Articulación escapulo humeral: Es la relación articular que existe entre la cavidad glenoidea de la escápula y de la cabeza del húmero, esta es una articulación que se clasifica como diartrósica triaxial, enartrósica, con posibilidad de movimiento en los tres ejes: transversal, sagital, vertical y planos: frontal, sagital y transversal, presentando una gran amplitud de movimientos para facilitar el trabajo de los miembros superiores, por lo cual es considerada como una de las articulaciones principales del organismo humano. 
Articulación humerocubital: Es una articulación diatrósica uniaxial del tipo troclear, sus movimientos se realizan a expensas del eje transversal y en el plano sagital, aunque puede llegar a ser biaxial. También se incrementa la permeabilidad de las membranas y el transporte de sustancias hacia y desde las distintas células (sustratos energéticos, 02, etc.).

En este movimiento se pone de manifiesto una anteversión del miembro superior izquierdo.

El cual se encuentra en un plano Sagital y un eje transversal.

\section{Plano sagital:}

Plano de orientación espacial que divide el cuerpo en dos mitades simétricas, derecha e izquierda.

Eje transversal:

Permite el desplazamiento de una estructura sobre el plano sagital.

\section{Clasificación de los huesos}

1. Los huesos por su forma se clasifican:

2. Largos Tubulares: fémur, tibia, peroné, cubito, radio.

3. Cortos Esponjosos: carpos, tarsos, vértebras.

4. Largos Esponjosos: costillas.

5. Anchos y planos: escápulas, huesos del cráneo.

6. Irregulares: etmoides, esfenoides.

\section{Clasificación de las articulaciones}

Sinartrósicas o inmóviles: son las articulaciones que no permiten desplazamientos y su función fundamental es la protección, se manifiestan en forma de sindesmosis, sincodrosis, sinostosis, ejemplos: las relaciones de los huesos del cráneo, la pelvis, etc.

Anfiartrósicas o semimóviles: esta articulación permite pequeños desplazamientos con una función fundamental mente de protección y sostén del organismo, ejemplo: la sínfisis del pubis, la membrana obturadora, las articulaciones intervertebrales, etc.

Diartrósicas, sinoviales o móviles: son aquellas que permiten una amplia gama de movimientos, por tanto, participan activamente en la ejecución de los movimientos del hombre en su actividad física, se clasifican en simples, compuestas, combinadas y complejas, 
ejemplo: la articulación escapulo humeral, la coxofemoral, etc. A subes se dividen en uniaxiales, biaxiales y poliaxiales de acuerdo a sus grados de movimientos y trayectorias posibles.

\section{Clasificación de las articulaciones diartrósicas}

Uniaxiales: Se desplazan en un solo eje de movimiento y generalmente permitan solo dos movimientos, ejemplos: flexión / extensión, pronación / supinación.

Biaxiales: Se desplazan a expensas de dos ejes de movimiento

Triaxiales: Se desplazan a expensas de tres ejes de movimientos e infinidad de trayectorias

Teniendo en cuenta las posibilidades de movimientos se subdividen en las siguientes categorías:

Trocleares o ginglimos: son articulaciones uniaxiales que permiten generalmente los movimientos de flexión y extensión, generalmente presentan accidentes óseos que limitan estos movimientos.

Trocoides: son articulaciones uniaxiales que se mueven en forma circular, con ligamentos anulares o cupulares que condicionan el movimiento.

Artrodias o planas: son articulaciones uniaxiales que permiten pequeños movimientos de desplazamientos a deslizamientos

Condíleas: son articulaciones biaxiales, donde una de sus caras articulares es convexa (cóndilo) y otra cóncava, en ocasiones presenta fibrocartílagos (meniscos) para hacer coherentes las caras articulares.

Enartrósicas o esferoides: son articulaciones triaxiales que permiten una amplia gama de movimientos y trayectorias de estos.

\section{Bioquímica}

\section{Zona de potencia máxima.}

- La duración del trabajo es de 20-30 segundos (anaerobio).

- El mecanismo energético fundamental: anaerobio alactácido.

- La principal reserva energética: CrP y ATP.

- El trabajo del corazón aumenta y la FC es de 180-190 puls/min. 
- Aumenta la glucosa en sangre.

- Consumo de $\mathrm{O} 2$ insignificante. Ventilación pulmonar mínima.

- Gasto general: 80 kcal.

- Deuda de O2 de 7-8 L.

- Incrementa la circulación.

- No se producen cambios significativos en la composición de la sangre.

- La recuperación ocurre de 30-40 min.

Por la Estructura del Movimiento se clasifica en un:

Movimiento acíclico:

No poseen una reiteración unida de ciclos, tienen una definida. Se caracterizan por la fuerza y rapidez (velocidad) máxima).

Cambios bioquímicos en músculos

- Aumento en el contenido de mioglobina.

- Aumento de la capacidad de oxidación de hidratos de carbono (glucógeno).

- Incremento en la oxidación de las grasas.

- Disminución en la producción de ácido láctico (aumento del umbral anaeróbico).

\section{Cambios bioquímicos en sangre}

- Aumento en el volumen sanguíneo

- Aumento en el número de hematíes (eritrocitos)

- Aumento en el número de leucocitos.

- Aumento en el hematocrito, hemoglobina y volumen celular.

- Aumento en el contenido de hierro.

- Aumento es en la actividad enzimática 
- Aumento es en la concentración hormonal: aumenta la concentración de adrenalina, $\mathrm{ADH}$, aldosterona, noradrenalina, cortisol, glucagón, disminuye la concentración de insulina.

- Variaciones en los niveles glicémicos: aumentan hasta $240 \mathrm{mg} / 100 \mathrm{ml}$ durante los trabajos anaerobios y disminuyen hasta $40 \mathrm{mg} / 100 \mathrm{ml}$ durante los trabajos aerobios.

- Variaciones en los niveles de ácido láctico: los niveles normales de 60-160 mg/100ml varían considerablemente hasta $250 \mathrm{mg} / 100 \mathrm{ml}$.

- Las reservas alcalinas tienden a disminuir hasta un 50\% durante los trabajos intensos $\mathrm{y}$ hasta un $12 \%$ en los trabajos de mediana intensidad.

Cambios bioquímicos en la orina

- La disminución de la tasa de la filtración glomerular.

- El aumento de la actividad simpática renal, la cual estimularía directamente en la célula tubular, la reabsorción de sodio.

- Volumen y excreción de agua y electrólitos

- ADH o vasopresina aumenta disminuyendo la tasa de filtración glomerular y el flujo sanguíneo renal.

- Disminuye la excreción urinaria de algunos electrólitos como sodio, cloro y potasio

- La estimulación por el ejercicio físico del sistema renina-angiotensina I, aldosterona.

Efectos entrenamiento anaerobio

- Incremento de la capacidad del sistema de los fosfágenos (ATP/PC)

- Un incremento de la actividad de las enzimas clave del sistema

- Un incremento de la actividad de las enzimas clave del sistema ATP/PC: ATPasa (ruptura de ATP).

- Mioquinasa (MK) (ADP-ATP).

- Creatinquinasa (CPK) (PC-ATP).

- Aumento de la capacidad glucolítica: fosfofrutokinasa 
- Aumento de la producción de lactato: (GLUCOGENOLISIS)

Efectos de la combinación del entrenamiento aeróbico y anaeróbico:

- La capacidad aeróbica no se ve afectada por el trabajo anaeróbico pero el entrenamiento aeróbico si afecta la capacidad anaerobia las fibras tipo I (fibras de contracción lenta ST procesan el ácido láctico producido y lo transforma en glucógeno para utilizarlo como fuente de energética, en la parte final de la carrera los músculos presentan elevada producción de ácido láctico y este provoca una acidificación en el músculo.

\section{Estados funcionales}

- Variaciones o cambios que caracterizan los diferentes estados del organismo y que se pueden manifestar antes durante y después de la actividad física tanto en entrenamiento como en competencia

- Son reacciones reflejas, antes, durante y después, del ejercicio provocadas por los requerimientos metabólicos.

- Pre-arranque: normal o de preparación competitiva, exaltación, apático

- Se produce antes de la competencia o el entrenamiento se puede presentar incluso varios días antes de competencia y en momentos preliminares de la competencia. $\mathrm{Su}$ base fisiológica son mecanismos reacciones reflejos condicionados.

- Específicas: el atleta sabe la intensidad del trabajo que se avecina en la carrera por su nivel de preparación o particularidades individuales.

- No específicas: Carácter y significado de la competencia, una clasificación a unos juegos olímpicos, campeonato mundial.

- Calentamiento: Complejo de ejercicios antes de una sesión de entrenamiento o competencia. Su valor fisiológico esta dado en que acorta la adaptación del organismo al esfuerzo.

\section{General y específico}

- Termorregulación

- Entrada al trabajo: Es el proceso mediante el cual el organismo se adapta paulatinamente a un nivel más elevado de la actividad. 
La gradual adaptación del organismo al esfuerzo se debe a la formación en la corteza cerebral de un foco de excitación. La adaptación se crea por un proceso de heterocronismo.

Heterocronismo. El aparato motor se adapta rápidamente por la elevada excitabilidad y la habilidad.

\section{Los órganos internos se adaptan más lentamente.}

Estado estable: Surge después de finalizada la entrada al trabajo y se comienza observar cuando el trabajo se extiende por no menos de 4 a 6 minutos, en ese momento se logra una estabilización del consumo de oxígeno y del funcionamiento de otros órganos y sistemas

Fatiga: Estado que se establece como consecuencia del trabajo y se manifiesta por una afectación de las funciones motoras y vegetativas, en la coordinación de estas, disminuyendo la capacidad de trabajo y la aparición de la sensación cansancio como resultado disminución de las reservas energéticas puede manifestar dolor por causa de la acumulación de ácido láctico hablando de los $800 \mathrm{mts}$ planos en la parte final

Recuperación: Adaptaciones al medio ambiente por medio del ejercicio y sus beneficios.

\section{Medio ambiente (altura)}

\section{En el sistema respiratorio}

- Hiperventilación

- Eliminación de una gran cantidad de $\mathrm{CO} 2$

- Aumento del PH corporal

- Difusión triplicada

- Aumento de la presión arterial

- Perdida de agua por evaporación

\section{En el sistema cardiovascular}

- El gasto cardíaco aumenta entre un $20 \%$ y $30 \%$.

- El flujo sanguíneo disminuye en la piel, riñones, y otros órganos, pero aumenta en el cerebro y corazón. 
- Aumento de vascularización del tejido muscular y óseo.

- Aumento del número y tamaño de los capilares.

\section{En la sangre}

- El aumento de la eritropoyesis

- Aumento de la eritropoyetina sanguínea

- Aumento en el número de hematíes por medula ósea

\section{En el sistema nervioso}

- Euforia

- Cefalea

- Mareos

- Insomnio

- Calambres musculares

Después de la inadaptación en la altura se producen los siguientes cambios:

- Aumento del número de capilares

- Aumento de las enzimas oxidativas

- Aumento del número de mitocondrias

- Incremento de la cantidad de glóbulos rojos

- Aumentan reservas alcalinas

Análisis Morfofuncional de la Conducta Espacial

Estructura Armónica del movimiento: Son aquellas que están dadas por la necesidad de estudiar los músculos como elementos asociados y no aislados.

- Cadenas o Acciones Musculares

Atendiendo a cómo se establecen (abiertas y cerradas) 
Abiertas para los miembros superiores

- Cadena Abiertas: Que no es más que la expresión estructurada y armónica del movimiento culmina con la libertad de acción mano digital.

Figura 1. Entrenamiento

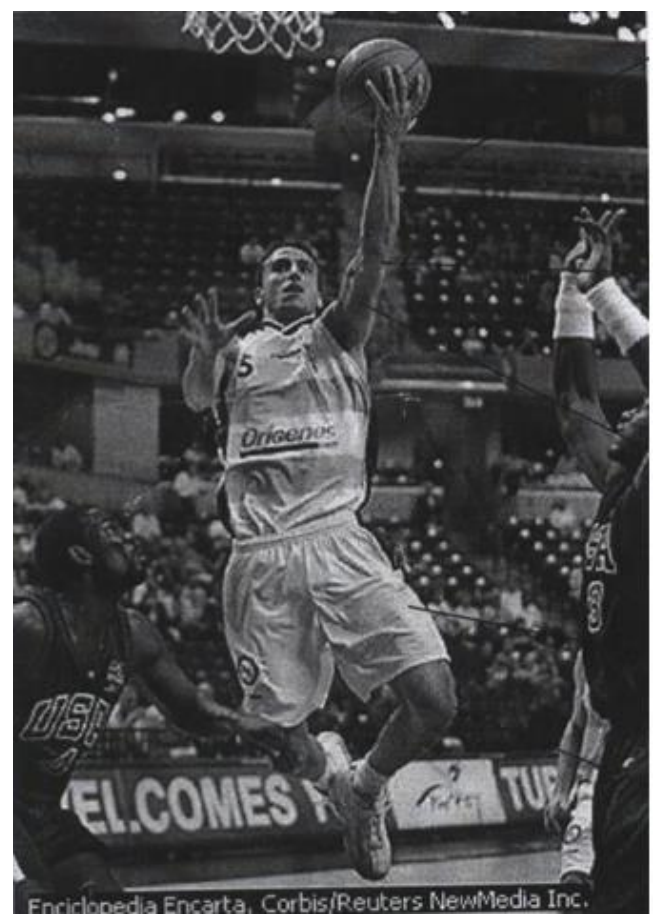

Fuente: Equipo Orleans.

\section{Principios del entrenamiento deportivo.}

Principios: Son postulados, normas, reglas que rigen un proceso y en este caso el del entrenamiento deportivo.

Son 5 principios que rigen el entrenamiento deportivo:

- Orientación y continuidad del entrenamiento.

- Aumento progresivo y máximo de las cargas.

- Cambios ondulatorios de las cargas.

- Carácter cíclico del proceso de entrenamiento.

- Unidad preparación general y preparación especial. 


\section{Orientación y continuidad del entrenamiento.}

El entrenamiento deportivo es un proceso ininterrumpido que se lleva a cabo por varios años en la preparación del deportista.

Sus características fundamentales están dadas por los aspectos siguientes:

- El proceso de entrenamiento transcurre a lo largo del año o muchos años seguidos, mantiene la orientación al perfeccionamiento en el deporte elegido.

- La influencia de cada ciclo de entrenamiento ulterior se materializa sobre la base de las huellas del anterior.

- Los intervalos de descanso entre los entrenamientos se mantienen en los límites que garantizan el establecimiento y el incremento de la capacidad de trabajo con la particularidad de que se permiten periódicamente la realización de entrenamientos con deudas parcial de restablecimiento.

Principio aumento progresivo y máximo de las cargas.

Tiene como esencia que las cargas deben ir cambiando poco a poco hasta llegar a la máxima carga y así poder adaptarse.

Ejemplo.

Paso uno se realiza el test de fuerza máxima.

Paso dos se planifica.

Paso tres se realiza el test de fuerza máxima y se ven los resultados si ha mejorado o no.

El cumplimiento de este principio debe responder a los siguientes aspectos:

- Las cargas físicas es necesario aumentarlas sistemáticamente.

- El aumento de las cargas y de las exigencias deben ocurrir de manera gradual.

- En el aumento de las cargas es necesario aplicar al organismo, periódicamente cargas máximas.

- La sistematización del aumento de las cargas debe ser lo más integral posible.

- Estudiar la magnitud de la carga empleada con el efecto fisiológico que se produce en el organismo. 
- Las cargas máximas están condicionadas a la preparación del atleta.

\section{Principio cambios ondulatorios de las cargas.}

Presupone que la carga no debe planificarse de forma rectilínea ni de forma escalonada ya que no se podría cumplir con la ley de la adaptación este principio recae en la ejecución de las cargas de forma ondulatoria.

En relación con este principio existen 3 tipos de ondas: las pequeñas, medias y grandes, que caracterizan la tendencia general de la dinámica de las cargas en los diferentes ciclos del entrenamiento.

Pequeñas.

Caracterizan la dinámica de las cargas en los microciclos.

Medias.

Representan la tendencia general de las cargas de unas cuantas ondas pequeñas en los límites de las etapas de entrenamiento.

Grandes.

Caracterizan la tendencia general de las ondas medias en los períodos de entrenamiento.

Principio carácter cíclico del proceso de entrenamiento.

Para mayor estudio se divide en ciclos lógicos; ciclos pequeños microciclos, ciclos medios mesociclos y ciclos grandes macrociclos.

En la actividad práctica, el principio de carácter cíclico se expresa por dos situaciones básicas:

- El contenido de entrenamiento deportivo es necesario cambiarlo de tal forma que contribuya al desarrollo de la capacidad de trabajo del atleta. Esto se logra por los cambios periódicos de todos los elementos durante el proceso de entrenamiento en los límites de un tiempo determinado.

- Todos los cambios se deben efectuar en los límites de ciertos tiempos, planificados con anticipación, que abarcan la preparación del deportista los cuales se definen de la forma siguiente: Macro, mesos y micros.

Principio unidad de la preparación general y la preparación especial. 
Este principio no indica que debe existir una estrecha dialéctica entre ambas preparaciones del periodo preparatorio ya que los elementos que se trabajan en la preparación general sirven de base para la preparación especial.

Tipos de adaptación.

Adaptación aguda, rápida o urgente.

Nos es más que la respuesta inmediata a la acción del esfuerzo ósea después de un ejercicio hay efecto en ello existen modificaciones del metabolismo en lo que se refieren las reservas energéticas y funciones metabólicas.

Adaptación crónica.

Existe un gran lapso de tiempo tiene como base la adaptación urgente y las huellas dejadas por las repeticiones del ejercicio, se relaciona con los cambios estructurales y funcionales que aumentan las posibilidades adaptativas de este se logra por la activación del aparato genético de células y la intensificación de síntesis de proteína.

\section{Conclusiones.}

- Mediante este trabajo se puede llegar a la conclusión que si aprendemos a conocer nuestro cuerpo, sus adaptaciones, sus cambios, sus distintos tipos de Sistemas como son: Sistema cardiovascular, Sistema Nervioso, etc., entonces seremos capaces de reflejar de forma productiva durante la ejecución de las diferentes técnicas deportivas, logrando así de esta forma una mejor salud y resultados deportivos.

\section{Referencias bibliográficas.}

CD Tutorial de Cultura Física.

Enciclopedia Encarta Baloncesto.

Pedro Luis de la Paz Rodríguez. Baloncesto (La ofensiva)

Dregomir V. Kirkov, Manual de Baloncesto.

Roberto Hernández Corvo, Morfología Funcional Deportiva.

Cayetano González Abin. Introducción y Desarrollo del Baloncesto en Güines.

Cardellá, L, Hernández, R. y col. "Bioquímica Médica”, Tomo I, II y III, Edit. Ciencias Médicas, C. de la Habana, 1999. 
Colectivo de Autores.CD Maestría Actividad Física en la Comunidad.ISCF”Manuel Fajardo,La Habana,2006.

Costill D.L., Wilmore J.H. "Fisiología del Deporte y del Ejercicio", Edit. Paidotrlbo, Barcelona, 1999

Fox E. “Fisiología del deporte” Edit. Panamericana, México, 1978.

Ganong W.F. "Fisiología Medica” 9ª Ed., Edit. El Manual Moderno, México,1988.

Guyton. A. Fisiología Humana. 6ta edición. Ed. Revolucionaria, La Habana, 1989.

Hernández. Corvo, R. Morfología Funcional Deportiva. La Habana. Ed.Cient Técnica, 1986.

Jurgen Weineck, Functional Anatomy in Sport. Chicago: Year Book Medical, 1986.

Katharine Well, Kinesiology Ed. Saunders, 5taedic. , 1971.

Lamb D.R. "Fisiología del Ejercicio", Madrid, 1985.

Lehninger, A."Bioquímica”, 2da. Edición, Edit. Revolucionaria. de la Habana, 1981.

León Oquendo, M. "Bioquímica: Bases para la Actividad Física”, Edit. Deportes, C.de la Habana, 2004.

López Chicharro J.- Fdez. Vaquero A., "Fisiología del Ejercicio", Edit. Medica Panamericana, Madrid, 1995.

Menshikov, V.V. y N.I. Volkov "Bioquímica”, Edit. Vneshtorgizdat, Moscú, 1990.

Prives, M. et al. Anatomía Humana Tomo I. Ed. Mir, Moscú, 6ta edic., 1984.

Sinélnikov, R.Atlas de Anatomía Humana. Tomo I. Ed. Mir, Moscú, 4ta edic., 1984,3t.

Tatárinov. Anatomía y Fisiología Humana. Ed. Mir, Moscú, 1980.

V.G. Elisev Histología.et al, Ed. Mir, Moscú. 1985.

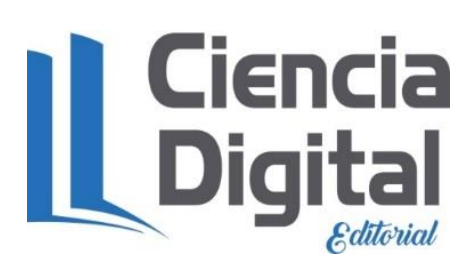




\section{PARA CITAR EL ARTÍCULO INDEXADO.}

González Pérez, E. R., \& González Paneque, A. (2018). Análisis de los factores morfológicos, bioquímicos, fisiológicos y biomecánicos que influyen en el tiro al aro en $\begin{array}{llll}\text { movimiento. } & \text { Anatomía } & \text { Digital, } & \text { 3(3), }\end{array}$ https://doi.org/10.33262/anatomiadigital.v1i3.1054

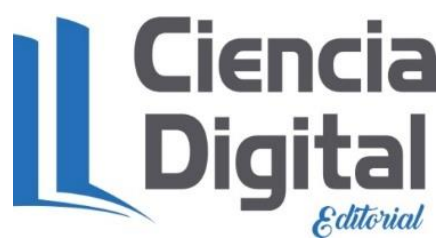

El artículo que se publica es de exclusiva responsabilidad de los autores y no necesariamente reflejan el pensamiento de la Revista Anatomía Digital.

El artículo queda en propiedad de la revista y, por tanto, su publicación parcial y/o total en otro medio tiene que ser autorizado por el director de la Revista Anatomía Digital.
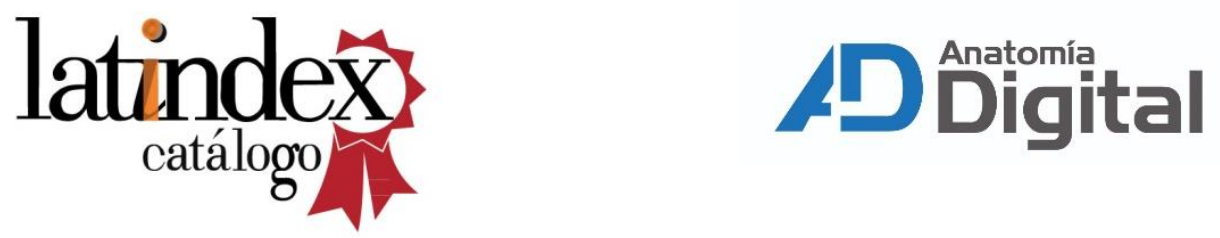